\title{
TEORÍAS DEL VALOR: COINCIDENCIAS Y DIVERGENCIAS EN LA ECONOMÍA Y LA ANTROPOLOGÍA SOCIAL
}

Juan José García del Hoyo*

Celeste Jiménez de Madariaga**

$\mathrm{D}$ esde la consolidación de la economía y la antropología hay cierta incompatibilidad entre ellas, debido quizá a diferencias metodológicas: la contraposición entre lo cualitativo y lo cuantitativo, que algunos autores simbolizan con el término $\mathrm{Q}^{2}$ para señalar que son los "dos extremos a lo largo del continuo de las ciencias sociales" (Bardhan y Ray, 2008, 427-428). Aunque en la antropología surgió una rama dedicada a temas "económicos", la economía suele omitir sus aportes. Por ejemplo, F. Knight, fundador de la Escuela de Chicago, en su polémica con M. Herskovits - quien acuñó el término "antropología económica"- hizo duras afirmaciones y proclamó que la economía era la única ciencia social que empleaba el método hipotético deductivo; aunque este episodio fue intrascendente porque la mayoría de los economistas se mantuvo indiferente ante una disciplina "dedicada a culturas primitivas" (Hamilton, 1991, 937).

Este artículo revisa las respuestas de ambas disciplinas al problema del valor, muestra su complementariedad y la necesidad del estudio interdisciplinario. Primero revisa la interacción entre economía y antropología económica y sus dificultades. Luego considera el concepto de valor, muestra los aportes de las principales escuelas de pensamiento económico y destaca las discrepancias entre las corrientes objetivistas y subjetivistas. Después muestra la influencia de la antropología social

* Catedrático de Métodos Cuantitativos para la Economía y la Empresa, Universidad de Huelva, España, [hoyo@uhu.es].

** Profesora titular de Antropología Social, Facultad de Humanidades, Universidad de Huelva, España, [celeste@uhu.es]. Fecha de recepción: 26 de marzo de 2014, fecha de modificación: 28 de julio de 2014, fecha de aceptación: 29 de octubre de 2015. Sugerencia de citación: García del Hoyo, J. J. y C. Jiménez M. “Teorías del valor: coincidencias y divergencias en la economía y la antropología social”, Revista de Economía Institucional 17, 33, 2015, pp. 109-131. DOI: http://dx.doi. org/10.18601/01245996.v17n33.05 
y la etnología en la visión institucionalista. Luego trata el concepto de "cosa útil" vinculándolo a las necesidades y a la idea de utilidad. Termina con unas conclusiones que destacan la convergencia entre ambas disciplinas en los últimos años.

\section{EL DIFÍCIL MARIDAJE ENTRE ECONOMÍA Y ANTROPOLOGÍA}

Aunque en un comienzo no se hacían estudios específicos de antropología económica, la etnografía clásica resalta la importancia de incluir los aspectos económicos para entender mejor los sistemas culturales, como atestigua la obra de Malinowski, Firth y Evans-Pritchard. En la formación de la antropología económica, a partir de la propuesta de Herskovits, hubo un debate entre dos corrientes contrapuestas: la formalista, para la cual los principios de la economía neoclásica se podían emplear para estudiar cualquier sociedad, y la sustantivista, que los rechazaba porque eran cuestionables en el estudio de las “sociedades primitivas". En medio de esta controversia, surgió una corriente de orientación marxista, integrada entre otros por $\mathrm{M}$. $\mathrm{Ha}$ rris, A. F. Wallace y M. Godelier, que proponía “analizar y explicar las formas y estructuras de los procesos materiales de cada sociedad [...] principalmente los 'modos de producción'y las 'estructuras económico sociales"' (Godelier, 1981, 13).

Ambas disciplinas se han ignorado mutuamente, como muestran algunos estudios sobre citación en revistas científicas, como el de Pieters y Baumgartner (2002) en revistas de ciencias sociales publicadas entre 1995 y 1997, o el de Pearson (2002), que examinó veintitrés revistas de economía publicadas entre 1955 y 2000. Pese a ese aislamiento relativo, en el campo de la economía se empezó a impulsar una visión interdisciplinaria en los años sesenta. Uno de los primeros trabajos en el que colaboraron economistas y antropólogos fue el de Devons y Gluckman (1964), que reconocieron la dificultad de cruzar las fronteras entre sus disciplinas y propusieron maneras de abordar problemas económicos desde la óptica de la antropología. Joy consideró inviable la aplicación de modelos antropológicos en economía y viceversa, sin utilizar métodos y variables de la otra disciplina, y subrayó la necesidad de mayor cooperación.

La distancia entre antropólogos y economistas obedece, entre otras razones, al tipo de sociedades que estudiaron inicialmente y a sus diferentes perspectivas (Hamilton, 1991, 938). Los economistas se ocupan de una sociedad donde predomina la economía de mercado, mientras que los antropólogos empezaron estudiando sociedades no mercantiles y consideraban inaplicable la teoría económica formal en 
esos contextos. Dalton, por ejemplo, señaló que la "economía primitiva" es diferente del "industrialismo de mercado", donde "toda persona se gana la vida vendiendo algo" $(1961,1)$. Aunque algunos principios económicos se puedan generalizar (la universalidad que defienden los economistas), hay que tener en cuenta las estructuras organizativas e institucionales de cada sociedad (la diversidad y las variables culturales de los antropólogos). Otros autores, como Cohen $(1974,114)$, afirman que es posible aplicar el instrumental económico al estudio de las "sociedades primitivas", y observan que las diferencias de método y el uso de técnicas matemáticas han sido los factores determinantes de la separación entre antropología y economía. Pryor (1977) utilizó técnicas econométricas para analizar datos de transacciones comerciales en un bazar y demostró que era posible usarlas en estudios de casos antropológicos.

E1 debate sobre la difícil relación entre economía y antropología aún perdura, e incluso se ha acusado a la economía de imperialismo ante las demás ciencias sociales; aunque algunos autores, como Booyens (1988), destacan la cercanía entre algunas corrientes teóricas, como el materialismo cultural y la economía convencional; otros proponen un maridaje entre ambas disciplinas, y hay quienes proponen unificarlas, dada su convergencia metodológica. Douglas (1973) invitó a aplicar métodos económicos en el estudio de instituciones culturales como el matrimonio, y Dalton (1978) mostró que la antropología económica hacía aportes a la economía. Otro ejemplo de cooperación es el estudio de poblaciones pequeñas en India de P. Bardhan, quien promovió el econ-anthro dialogue (Bardhan y Ray, 2008). Otros aspectos que solía tratar la antropología social (como la familia y el parentesco) se han analizado conjuntamente con la economía, como en el libro de Haddad et al. (1997). La preocupación por los "países en desarrollo", la problemática de los alimentos y el aprovechamiento de los recursos han suscitado intentos de síntesis, como el trabajo de Harriss (2002) sobre la necesidad del estudio interdisciplinario de los procesos de desarrollo, y el de White (2002) sobre la necesidad de combinar métodos cuantitativos y cualitativos en el análisis de la pobreza y la desigualdad.

Lipton plantea que esta difícil cooperación no obedece a diferencias metodológicas sino a su objeto; mientras que "la economía se interesa principalmente por los resultados, la antropología se interesa en los procesos" (1992,1541). Los economistas elaboran modelos -a veces simples cajas negras- para hacer predicciones y poco se preocupan por el análisis explicativo, el cual es fundamental para los antropólogos. Eso no significa que los economistas no usen técnicas y modelos 
econométricos para descubrir relaciones, sino que su objetivo es la predicción y la simulación de escenarios. Para algunos autores esta es la razón de la incomprensión entre ambas ciencias, no las diferencias metodológicas que son, más bien, resultado de tradiciones académicas (Bardhan, 1989, 10-11).

Algunos trabajos analizan problemas económicos con el método etnográfico, como el de Buckley y Chapman (1996). Otros autores muestran que la antropología ha contribuido a entender mejor las relaciones de poder -en la sociedad, en mercados, en ramas industriales y entre usuarios de bienes comunes- en cuanto están asociadas a representaciones simbólicas y no solo a aspectos puramente materiales (Rao y Walton, 2004). Y en una dirección contraria, hay autores que unen el método etnográfico y el de la economía experimental, y proponen que este último es el más conveniente para contrastar teorías antropológicas (Ensminger, 2002). Henrich et al. (2004) unen estos dos métodos para estudiar micro sociedades tradicionales y hacer comparaciones transculturales.

En vista de estos antecedentes, pretendemos ilustrar el potencial maridaje entre economía y antropología revisando el desarrollo de la teoría del valor.

\section{EL VALOR COMO CONCEPTO}

Aquí se enfrenta la difícil tarea de definir el "valor", noción que tiene significados distintos en cada ciencia social. Primero mencionamos algunas definiciones simples, como la de Morris, para quien el estudio de los valores es "la ciencia del comportamiento preferencial" (1956, 12), y la de Perry, que lo define como "cualquier objeto de interés" $(1950,115)$, la cual se discutió en un seminario sobre la teoría general del valor realizado en 1951, donde Kluckhohn señaló:

un valor es una concepción de lo deseable, explícita o implícita, distintiva de un individuo o característica de un grupo, que influye en la selección de los modos, medios y fines de la acción [...] Esta definición toma como puntos de partida a la cultura, al grupo y a la relación del individuo con la cultura y a la posición en su grupo $(1951,395)$.

\section{Para Gregory}

Los valores son esas cadenas invisibles que vinculan las relaciones entre cosas con las relaciones entre personas; y el valor es expresión de una relación antagónica de poder entre personas histórica, geográfica y antropológicamente localizadas (1997, 33).

Es claro que en estas definiciones antropológicas hay una interacción entre factores culturales y económicos, y parecen contraponerse a 
las que provienen de la economía, aunque esto supondría que "los procesos económicos no son procesos culturales” (Díaz, 2007, 123). De la definición de Kluckhohn cabe destacar tres elementos: los valores sociales son esenciales para la integración y la permanencia del cuerpo social; el lazo entre ideas y valores, es decir, entre aspectos cognitivos y normativos; y, finalmente, la organización jerárquica de los valores en virtud del grado de deseabilidad del objeto, es decir, que "en la motivación y en el valor influyen la cultura y la historia vital del individuo" (Kluckhohn, 1951, 398 y ss.). Foster (2011) señala que el valor siempre implica una comparación, bien sea en términos morales, semióticos o económicos. De los dos primeros no se suelen ocupar los economistas, pues "se han dejado en gran parte a antropólogos, sociólogos o filósofos” (Graeber, 2006, 439).

A continuación revisamos las respuestas que la economía y la antropología han dado al problema del valor, teniendo en cuenta que "el aspecto humano, en el sentido antropológico del término, es esencial para entender los fenómenos sociales" (Castaingts, 2002, 12).

\section{LOS PRIMEROS APORTES}

La utilidad o valor de uso de un bien es la satisfacción que proporciona a una persona; el valor de cambio es su precio en dinero u otros bienes. Una teoría del valor explica por qué los bienes tienen valor de uso y cómo se forman o determinan sus precios. Aristóteles dio por sentado el valor de uso y solo analizó el precio corriente, que para él dependía del costo de producción. Agustín de Hipona añadió el concepto subjetivo de necesidades. Alberto Magno definió el valor de cambio como precio relativo e introdujo la equivalencia entre precio justo y restitución de los costos y el trabajo. Esto dio lugar a una separación más nítida entre valor de uso y valor de cambio, y a dos interpretaciones del valor: la subjetivista, basada en la satisfacción de las necesidades, y la objetivista, en los costos de producción. Tomás de Aquino defendió esta última y planteó la equivalencia entre precio justo -valor de uso- y costos de producción, incluido un "lucro moderado" para retribuir el riesgo de los comerciantes. Para J. Buridan la satisfacción de las necesidades era la medida real de los bienes; y para Bernardino de Siena el valor de uso dependía de tres elementos: virtuositas, raritas y complacibilitas, aunque pensaba que la utilidad era intrínseca al bien; él resolvió la paradoja del valor: el hecho de que algunos bienes con alto valor de uso tienen poco valor de cambio, y otros de poco valor de uso tienen alto valor de cambio, introduciendo el concepto de escasez (raritas). 
La escolástica española criticó el objetivismo. Francisco de Vitoria identificó el precio justo con el precio de mercado, criticó a quienes lo asociaban con el costo y lo vinculó a la "común estimación”. Luis de Molina examinó de modo más preciso el problema de la valoración y clasificó los precios en legales y naturales; estos últimos son los que tienen las cosas por sí mismas y dependen del "afecto y estimación" que se les concede, de modo que el precio natural fluctúa dentro de ciertos márgenes, según las estimaciones subjetivas.

Algunos autores italianos hicieron otros aportes. Davanzati estudió el valor del dinero, planteó la paradoja del valor y se acercó a su solución mediante la escasez. Su principal aporte fue considerar la utilidad como algo propio del individuo y no de la cosa deseada, como sostenían los escolásticos. Galiani argumentó que la escasez (rarità) dependía de la utilidad (utilità) y que esta variaba según el uso; en la formación de precios añadió un matiz adicional: la influencia de la cantidad de trabajo (fatica) en la oferta y del consumo en la demanda. Verri elaboró una verdadera teoría de los precios. Resolvió la paradoja del valor mediante la escasez, y estableció que la utilidad y la escasez determinan el precio. En cuanto a la demanda precisó que "la necesidad no es sino la estimación que se hace de la mercancía que se desea en parangón con aquella que se quiere ceder" (1820, 26). Genovesi refinó la interpretación subjetiva del valor y planteó "que nuestras necesidades son el origen del valor de todas las cosas, y el precio de estas es su poder para satisfacer nuestras necesidades" $(1786,10)$. Antes de Menger, clasificó las necesidades humanas, pero su análisis del valor se limitó al valor de cambio y a la formación de precios en libre competencia y en monopolio.

Turgot retomó el concepto de utilidad relativa de los autores italianos y argumentó que el valor era en parte una propiedad intrínseca del objeto, aunque dio primacía a los aspectos subjetivos. Condillac, por su parte, señaló que una cosa es útil cuando satisface una necesidad y que su valor es la estimación atribuida por los usos que se le puede dar, mientras que el precio es el valor relativo frente a otras cosas, y depende de su abundancia relativa y de la concurrencia de compradores y vendedores.

\section{LA ECONOMÍA CLÁSICA: OBJETIVISMO RADICAL}

Petty se anticipó a los clásicos en lo relativo a la división del trabajo y a la teoría del valor, al que relacionó con la tierra y el trabajo. Locke señaló que la contribución de la tierra al valor era menos relevante que la del trabajo, pues "si no fuera porque el trabajo nos hubiese 
proporcionado estos productos, nuestro pan, nuestra bebida y nuestros vestidos no serían más que bellotas, agua, hojas o pieles" $(2000,68-$ 69). Cantillon distinguió dos componentes del valor de cambio: el "precio o valor intrínseco"-relacionado con el precio justo- y el que es determinado por la necesidad, al que llamó "precio de mercado". E1 primero es "la medida de la cantidad de tierra y de trabajo que intervienen en su producción" (1950,28), que también "depende del humor y la fantasía de los hombres y del consumo” (ibíd., 29).

Adam Smith diferenció entre valor de uso y valor de cambio y se centró en este último, que "para la persona que posee [un bien] es igual a la cantidad de trabajo ajeno que con ella quede habilitado a granjear" (1794, 49). Buscaba establecer una unidad de cuenta homogénea y estable ante las fluctuaciones de la moneda y su idea del precio natural era semejante a la de Cantillon, pero añadió que el precio de mercado fluctúa alrededor de este en el corto plazo y se ajusta automáticamente por obra del mercado, guiado por la mano invisible que asigna eficientemente los recursos. Su teoría del precio olvida que los costos de producción se establecen en los mercados de factores productivos y, por tanto, su argumento es circular.

Ricardo también propuso una teoría de los precios basada en los costos de producción: "la utilidad no es la medida del valor de cambio, aunque es absolutamente esencial para este” $(2004,9)$, pues es una condición necesaria para que un bien tenga valor, el cual está determinado por la cantidad de trabajo requerida para producirlo y por la escasez; además de incorporar las ganancias del capital, valoradas por el trabajo necesario para su acumulación.

Malthus hizo tres distinciones: el valor de uso propiamente dicho o "utilidad intrínseca", el valor nominal de cambio expresado en metales preciosos y el valor de cambio intrínseco que hace equivalente el resultado "de la oferta comparada con la demanda" $(1946,51)$, que puede ser independiente del costo de producción en "las mercancías de mayor tráfico", lo que niega la existencia de una medida objetiva del valor de cambio (ibíd., 59).

Say se opuso a todo cálculo objetivo que no tuviera en cuenta la utilidad y estableció un vínculo entre valor de uso y valor de cambio; este último se fija "en cada lugar conforme al valor que le dan sus costos de producción, a condición de que la utilidad que se le atribuye dé origen al deseo de adquirirlos" (2000,281). Enlazó la teoría del valor a la distribución mediante la retribución a los factores productivos: la renta de la tierra, los salarios del trabajo y el interés del capital. Say abrió el camino a la teoría moderna al considerar el valor de un bien 
como resultado de su utilidad, expresada en la demanda, y del costo de producción, reflejado en la oferta.

Para J. S. Mill la teoría del valor estaba bien establecida ${ }^{1}$, pero hizo una distinción entre valor de cambio o "capacidad general de compra" y precio corriente, "su valor en dinero" (1951, 387), y añadió que el primero era el límite superior del segundo. Su visión del precio es casi idéntica a la de Ricardo: las mercancías tienen un valor de cambio natural determinado por el costo de producción y un precio determinado por la concurrencia de la oferta y la demanda, que tienden a ser iguales en el largo plazo.

Marx argumentó que una mercancía solo podía tener valor de cambio si poseía valor de uso, es decir, si satisfacía necesidades humanas, así brotaran "del estómago o de la fantasía” (1988, 3). Hasta aquí su visión era más subjetivista que la de Smith, Malthus o Ricardo, pero inconclusa: "ningún objeto puede ser un valor sin ser a la vez un objeto útil. Si es inútil, lo será también el trabajo que encierra; no contará como trabajo, no representará, por tanto, un valor" (ibíd., 8). Luego se apartó del subjetivismo y elaboró su teoría del valor trabajo, según la cual los precios giran alrededor del valor y este consiste en "la cantidad de trabajo socialmente necesario" para producir un bien, la cual varía con la tecnología y la cualificación de los trabajadores. Y explicó la distribución recurriendo al concepto de plusvalía, la idea de que los capitalistas no pagan a los trabajadores todo el valor de su trabajo sino el salario necesario para su subsistencia.

Con Marx se cierra el ciclo de la economía clásica, el cuerpo doctrinal que es el fundamento de las teorías modernas, aunque se podría decir que los economistas clásicos no formularon una teoría del valor sino una teoría circular de los precios.

\section{EL MARGINALISMO Y LA ECONOMÍA NEOCLÁSICA}

El subjetivismo triunfó con la revolución marginalista. E1 concepto de utilidad marginal surgió cuando los economistas se interesaron en los aspectos generales de la ciencia y empezaron a utilizar el cálculo diferencial, encabezados por Jevons, Menger y Walras. El objeto de la ciencia económica se redujo a "maximizar la felicidad proporcionando placer con el menor costo posible de dolor" (Jevons, 1871, 27); y, siguiendo las ideas de Bentham, la utilidad -la capacidad de un bien para producir placer o evitar un mal- se convirtió en una magnitud

\footnotetext{
1 "Afortunadamente, no queda nada que aclarar en las leyes del valor, ni para los escritores actuales, ni para los del porvenir: la teoría está completa" (Mill, 1848, 386).
} 
cuantificable; y la teoría del valor pasó a basarse en el concepto de utilidad marginal: cuantas más unidades de un bien se consuman, menor será la utilidad que proporciona, y su valor estará dado por la utilidad que proporciona la última unidad consumida.

Jevons y Walras emplearon una escala cardinal de preferencias -cardinalismo introspectivo-, y Menger una escala ordinal, más general y precisa, pero no utilizaron el término "utilidad marginal". Jevons distinguió entre "utilidad total", "grado de utilidad" y "grado final de utilidad", la cual era la utilidad de la última adición de una cantidad infinitamente pequeña del bien. Walras usó el término rareté, "intensidad de la última necesidad satisfecha" $(1977,119)$. Y Menger diferenció los niveles de necesidad de un bien, con su grado de utilidad respectivo, y sostuvo que su valor "es igual a la importancia de la necesidad menos importante entre las que puede satisfacer" (1871, 131). Aunque los marginalistas hacían la distinción entre valor de uso y valor de cambio, señalaron que esos términos eran ambiguos y los sustituyeron por los de utilidad y precio, respectivamente.

Para Walras y Menger el costo de producción no determina los precios. Para Walras los determinan su utilidad -que se refleja en la demanda-y su escasez relativa -que se refleja en la oferta- y no el costo de los servicios productivos, el cual es determinado a su vez por la demanda y la oferta en el mercado de factores. Menger introdujo un matiz esencial: esta determinación no es simultánea y se basa en expectativas, los precios de los bienes de orden inferior son determinados por las condiciones actuales del mercado, y los de orden superior -factores productivos- por las expectativas sobre el futuro, es decir, por los precios esperados de los bienes que contribuyen a producir. Por tanto, explican la formación de precios mediante un mecanismo distinto al de la teoría clásica: en vez de que tengan una relación objetiva con los costos de producción postulan una relación subjetiva entre bienes y estados de conciencia, y como estos son rasgos invariantes de la naturaleza humana "el principio era válido para cualquier clase de sociedad” (Dobb, 1937, 26).

\section{LA ECONOMÍA NEOCLÁSICA}

El desarrollo de la economía neoclásica se basó en la obra de Marshall, quizás, el economista más influyente en la difusión de la ciencia. La economía era para Marshall, como para los marginalistas, la ciencia de lo útil y lo escaso, escasez circunscrita a las mercancías que se pueden consumir. Recurrió a la teoría de la utilidad marginal para deducir la curva de demanda, y a las funciones de costos para la curva de oferta, 
y sentó así las bases de la microeconomía convencional: "la utilidad marginal de una cosa, para una persona, disminuye con cada nuevo aumento de su oferta" $(1890,155)$. Pero mientras que señaló que la demanda determina los precios a corto plazo, en su afán de completar la teoría clásica mantuvo que en el largo plazo los determinaban los costos de producción y, en el mediano plazo, la conjunción de la oferta y la demanda sustentada en los costos y en la utilidad marginal. Como Bentham, consideró que la utilidad era agregable y comparable a través del dinero, de modo que el precio medía la utilidad marginal y se podían hacer comparaciones interpersonales. Su visión de la economía -Economía, como la denominó, contrapuesta a la Economía Política-abandonó la línea de Menger y Walras y retomó los costos de producción. Con el concepto de excedente del consumidor sentó las bases de la teoría del bienestar que desarrolló formalmente su discípulo A. Pigou, teoría que permite justificar políticas redistributivas basadas en impuestos progresivos para compensar las deseconomías externas reflejadas en la divergencia entre costos privados y sociales, así como la intervención estatal ${ }^{2}$. Pigou consideró que la utilidad marginal era aditiva, que el bienestar social se podía representar como la suma algebraica de las utilidades individuales y que la equidad distributiva implicaba maximizar la utilidad social.

Pareto (1945) adoptó el concepto de equilibrio general de Walras y elaboró una teoría del consumidor en la que sustituyó la utilidad marginal por las preferencias. Empleó las curvas de indiferencia introducidas por Edgeworth ${ }^{3}$, y propuso dejar de medir la utilidad con escalas cardinales y centrarse en las preferencias ordinales ${ }^{4}$. Criticó la teoría clásica del valor y su identificación del costo de producción con el precio o valor, pues este se determina por "la oposición entre los gustos y los obstáculos" $(1945,184)$, es decir, por la utilidad (ophélimité) y por los costos. En su desarrollo del equilibrio general, que formaliza la noción de la mano invisible, justificó la desigualdad en la distribución del ingreso defendiendo a ultranza los mecanismos de mercado que dan lugar a un óptimo social, y rechazó la injerencia

${ }^{2}$ Aunque sus ideas pronto fueron criticadas por economistas de Chicago, como F. Knight, quien negaba el papel del Estado en la redistribución, y para quien no existían fallas de mercado sino que la distribución de la propiedad era inadecuada.

${ }^{3} \mathrm{Su}$ obra enlaza con el cálculo hedonista de Bentham, pero ahora basado en el cálculo diferencial: "la concepción del hombre como una máquina de placer puede justificar y facilitar el empleo de términos mecánicos y el razonamiento matemático en la ciencia social" (Edgeworth, 1881, 15).

${ }^{4}$ Pareto y Fisher abrieron el camino para despojar a la microeconomía de todo atisbo utilitarista; las curvas de indiferencia permiten construir una teoría del consumidor basada tan solo en escalas de preferencia, sin justificarlas por la utilidad. 
estatal en la economía. Pero siguió a Walras y defendió la determinación de la oferta por los costos de producción.

La escuela austriaca, heredera de Menger, mantuvo en cierta medida este razonamiento. Böhm-Bawerk, definió el valor como "la importancia que adquiere un bien [...] al reconocerle una condición de utilidad que de otra manera no hubiese tenido para contribuir al bienestar de un individuo", siendo necesario, además, que el bien sea escaso "en comparación con la demanda de bienes en cuestión" $(1959,129)$. El precio no mide la utilidad marginal sino que refleja la interacción de la utilidad marginal de las personas, que delimita el rango de variación de los precios. Así, los costos no influyen en los precios, sino que son su consecuencia, aunque Böhm-Bawerk evitó hablar de expectativas y mantuvo la simultaneidad neoclásica.

Wieser entendió el "valor natural" como un fenómeno neutral independiente del régimen económico, que depende de la distribución del ingreso entre las clases sociales, de modo que, en una situación distante de la equidistribución, los bienes o servicios dotados de poca utilidad social pueden tener gran valor, y viceversa. El precio es determinado por ese valor natural, junto con la distribución del poder adquisitivo, los errores, fraudes y coacciones.

Von Mises interpretó la utilidad como "la importancia atribuida a una cosa por su supuesta capacidad para suprimir una incomodidad humana" $(1980,195)$, a la que relacionó con el "valor de uso subjetivo", mientras que el "valor de uso objetivo" es la relación entre una cosa y los efectos que produce. Aisló el concepto de utilidad de otras consideraciones y señaló que "el valor [de uso subjetivo] nada tiene que ver con palabras o doctrinas. La propia conducta humana, exclusivamente, engendra el valor" (Mises, 1968, 159).

\section{LA CRÍTICA INSTITUCIONALISTA}

La economía institucional surgió en Estados Unidos con la obra de Thorstein Veblen, John Commons y Wesley Mitchell, y tuvo un papel predominante hasta la difusión de la teoría keynesiana y de la econometría en la década de 1940. Para estos autores los precios son convenciones sociales reforzadas por los hábitos e incardinadas en instituciones concretas, que dependen de las ideas aceptadas. Por ello una explicación de los precios supone una teoría de las instituciones, del papel de los hábitos y de las expectativas. Veblen criticó la corriente neoclásica, por recurrir al cálculo hedonista de la utilidad proveniente de Bentham y Mill, por su inadecuada explicación del comportamiento económico y su visión estática: "la teoría de la utilidad marginal 
[...] no ofrece explicación alguna de ningún tipo de cambios porque solo se ocupa del ajuste de los valores en una situación dada" (Veblen, $1909,620)$, sin tener en cuenta aspectos esenciales como el cambio técnico, la cultura empresarial y las normas e instituciones que regulan la actividad económica. Así, por influencia de antropólogos como Morgan, Veblen consideró relevantes los elementos culturales que para la economía neoclásica solo son parte del escenario donde se desarrolla la acción. Mitchell insistió en que era necesario tener en cuenta los aportes de la etnología y la antropología al conocimiento de la naturaleza humana.

La economía institucional, la economía neoinstitucional y la antropología confluyen en su común interés por la acción colectiva, el papel de la tecnología y el cambio social. Todos los antropólogos económicos, salvo los formalistas más extremos, comparten principios de los institucionalistas. Como recuerda Hamilton (1991, 939-943), la influencia de Veblen en Herskovits y Sahlins fue determinante, y las teorías de la reciprocidad de Malinowski son el eje de la teoría del valor del institucionalismo radical (Hamilton, 1965, 241).

\section{ABANDONO DEL CARDiNALISMO UTILITARISTA}

E1 concepto de utilidad, que la corriente marginalista formalizó en funciones cardinales de utilidad, llevó a Pigou a justificar la intervención del Estado para lograr el máximo bienestar mediante medidas redistributivas. Pero L. Robbins argumentó que la utilidad no se puede medir porque es subjetiva e individual, y así no es posible un análisis científico de los efectos de las medidas redistributivas. Solo se podría desarrollar una teoría científica del bienestar si la utilidad se concibiera como una escala de preferencias, y se liberara a la economía de toda proposición normativa. El uso de las curvas de indiferencia llevó a que algunos economistas de Cambridge abandonaran la idea de que "existe alguna medida cuantitativa adecuada de la utilidad o la satisfacción” (Hicks, 1939, 18) e intentaran "purgar y rechazar todos los conceptos afectados de utilidad cuantitativa", sustituyendo la utilidad marginal decreciente por la convexidad de las curvas de indiferencia y la relación marginal de sustitución.

Con base en el trabajo de Slutsky, Hicks y Allen replantearon la teoría del consumidor-ordinalismo introspectivo- recurriendo a una escala ordinal de preferencias donde las curvas de demanda prescinden de la utilidad aditiva y solo son de carácter ordinal. Pero este esquema, basado en la comparación de pares de mercancías mediante curvas de indiferencia, es " $\tan$ introspectivo e inobservable como [...] el concepto 
de comparaciones cardinales entre utilidades marginales"(Blaug, 1992, 142), y se basa en dos supuestos psicológicos no universales: que los individuos actúan por introspección y que siempre buscan maximizar. El único aporte relevante de esta línea de pensamiento fue el concepto de relación marginal de sustitución y la descomposición de los efectos ingreso y sustitución en las curvas de demanda, pues cuando Hicks desarrolló totalmente sus argumentos, Samuelson ya había publicado los rudimentos de un nuevo paradigma -el ordinalismo conductista- con la teoría de las preferencias reveladas y un menor número de supuestos.

Samuelson recurrió a principios axiomáticos para justificar la teoría neoclásica del consumidor. Lo que se conoce no son sus preferencias sino las elecciones que ha hecho, y la utilidad es inadecuada en una teoría científica porque es imposible su verificación empírica (1938, 61), así como la idea de la tasa marginal de sustitución, y propuso elaborar una teoría que partiera del comportamiento observable. Pero, a pesar del formalismo matemático y del ambicioso objetivo, "estos teoremas no tienen nada que el economista pueda tomar en su intento de explicar la complejidad del mundo real” (Mishan, 1961, 1). Esta teoría, así como las del comportamiento del consumidor de Debreu y de la elección social de Arrow, en su afán de depurar todos los postulados irrelevantes, es "un ejemplo de cómo extraer el mínimo de resultados del mínimo de suposiciones" (Lancaster, 1966, 132). No obstante, las funciones de bienestar social de Arrow permiten obtener funciones de utilidad social -elección social- a partir de las preferencias individuales.

\section{REGRESO A POSICIONES OBJETIVISTAS}

Para Keynes la elección individual era resultado de factores objetivos y subjetivos -“características subjetivas y sociales que determinan cuánto se gasta” $(1998,142)-$, por lo que era imposible analizar el comportamiento social como agregación de elecciones individuales. Propuso una teoría del consumo que partía del comportamiento social observable y daba a los hábitos, reglas y convenciones un papel determinante, como los institucionalistas ${ }^{5}$. Sraffa (1960) criticó la teoría neoclásica, subrayó que la economía se debía basar en mag-

\footnotetext{
${ }^{5}$ Llegó incluso a afirmar: "la fuerza de todos estos motivos variará mucho conforme a la estructura institucional y económica [...], de acuerdo con las costumbres asociadas a la raza, educación, convenciones sociales, religión y principios morales vigentes, de acuerdo con las esperanzas y la experiencia del pasado, con la escala y la técnica del equipo de capital y de acuerdo, por último, con la distribución de la riqueza y niveles de vida" (ibíd., 143-144).
} 
nitudes observables y medibles y en hipótesis verificables, y evitar el concepto de utilidad. Trató de rehabilitar a Ricardo y Marx y sus análisis del proceso productivo, y argumentó que los precios relativos son independientes del patrón de consumo y de la demanda. El valor económico es un resultado social, de la estructura de clases concreta, del nivel tecnológico y de la manera de organizar la producción. En su teoría, los costos de producción son la base de los precios y determinan la distribución entre factores productivos, siguiendo a Ricardo. Parte de una economía de subsistencia, que refleja la influencia del antropólogo R. Firth ${ }^{6}$, y aumenta su complejidad hasta demostrar que hay una relación inversa entre salarios y beneficios, usando como unidad de cuenta o "patrón invariable" las cantidades físicas de mercancías "básicas". La obra de Sraffa renovó el interés por el pensamiento económico de Ricardo y Marx, y sus seguidores concuerdan con los neoinstitucionalistas en su crítica al eclipsamiento neoclásico de la teoría del valor (Garnett, 1999).

\section{ELACERCAMIENTO ENTRE LA ECONOMÍAY LAANTROPOLOGÍA}

Una escuela más reciente, el neoinstitucionalismo, se acerca de modo explícito a la antropología al subrayar el papel de las instituciones sociales. Su análisis del valor se centra en los costos de transacción, con un objetivismo neo ricardiano. Al costo de producción (que incluía el costo del capital, el trabajo y la tierra) añade los costos de transacción, que incluyen el costo de uso y el de la información requerida para garantizar el intercambio y superar la asimetría existente en todo intercambio. Estos costos suponen la normatización de los derechos y la institucionalización del valor, es decir, un "marco institucional". La importancia de las instituciones sociales en la organización de la economía, tanto en la economía doméstica como en comunidades más amplias, había sido señalada por los antropólogos. En 1922, Malinowski mostró que en algunas "sociedades primitivas" se institucionaliza el valor que se atribuye a las cosas (objetos suntuosos y de primera necesidad), y que el intercambio toma la forma de ceremoniales ritualizados ${ }^{7}$. Hamilton, un institucionalista radical, extendió esa idea a las sociedades modernas, donde el aspecto ceremonial recíproco

${ }^{6}$ Como señalan Kurz y Mondadori (2010, 202), Sraffa leyó y anotó a Firth (1929), y también consideró que el comunismo primitivo era una fantasía. Wittgenstein dijo que Sraffa le mostró la manera antropológica de ver las cosas Sen (2003, 1242).

${ }^{7}$ En su conocida obra sobre los trobriand, Malinowski (1922) mostró que en esa sociedad se había institucionalizado el intercambio económico, y prevalecía una forma de pago en especie a los familiares y una ceremonial (kula). 
del intercambio se reduce. Aunque el intercambio técnico de bienes y servicios, fruto de la división del trabajo y de la especialización, va acompañado de intercambios financieros ceremoniales en los que el dinero es un medio formal para autenticar el intercambio. C. Ayres y J. Foster mostraron que en las instituciones de la sociedad moderna aún pervive cierta ritualización de la economía, a la que entienden como un proceso evolutivo de cambio tecnológico, al cual se vincula el valor. Señalan dos funciones de las instituciones: la función instrumental, para el intercambio de objetos y tecnologías, y la función "ceremonial", que mantiene o consolida el estatus social y las relaciones de poder (Tool, 1977).

Para los neoinstitucionalistas, las instituciones garantizan y organizan los intercambios determinando los costos de transacción. Las instituciones varían según el tipo de sociedad, de las más simples a las más complejas, de las más homogéneas a las más heterogéneas, y dan lugar a distintas formas de intercambio, así como a productos con gran diversidad de atributos. En su análisis diacrónico del proceso de institucionalización, North (1990) distingue el "intercambio personalizado", que ha predominado históricamente y se caracteriza por la producción a pequeña escala y el comercio local, donde no se necesita una tercera parte que garantice los acuerdos; y el "intercambio impersonal”, en el que la producción a gran escala, los mercados distantes y las transacciones impersonales requieren instituciones complejas y un tercero que asegure su cumplimiento.

En la antropología, el concepto de valor varía entre las teorías basadas en la estructura y la organización social y las que se centran en aspectos del comportamiento. Perry $(1930,490)$ distingue dos características; una que alude a lo bueno, lo correcto, lo que debe ser, que implica decisión y preferencia y pierde su significado distintivo por el uso común, y la otra a su carácter razonable, pues de otro modo reflejaría un intento sutil de imponer deseos a los demás.

Graeber $(2001,2006)$ muestra que ha habido tres etapas en el estudio de la noción de valor. La primera, a finales de los años cincuenta, en la antropología social británica y en la antropología cultural estadounidense. El estructural-funcionalismo británico, encabezado por Radcliffe-Brown, estudió el valor desde un enfoque sistémico que concebía la sociedad como un organismo cuyas partes (instituciones) ayudan a mantener el orden social. En Estados Unidos se destacan la obra de Kluckhohn, que hizo un estudio comparativo del valor basado en su trabajo de campo en Nuevo México, en el que mostró que hay diferencias en los sistemas de valores de los distintos grupos sociales. 
Para él, los valores son "concepciones de lo deseable", aunque lo deseable no es simplemente lo que la gente quiere, pues los deseos están condicionados por el contexto cultural y, dependiendo de la sociedad, se desea el éxito, la armonía, la pureza, etc. El problema es comparar esos deseos pues sus significados y correspondencias en términos de valor no son iguales. Por ello, propuso un nivel menos abstracto, "las orientaciones de valor", que vincula el valor al conocimiento, lo que se supone es bueno y evita la frustración y el malestar. Esta comparación parecía ser más fácil que la de utilidades marginales, pero Kluckhohn fracasó en el intento.

En la segunda etapa, a comienzos de los ochenta, se elaboraron modelos estructuralistas aplicados a la idea de valor, en los que este se inscribe en un sistema abstracto de reglas y significados, y existe aparte de cualquier expresión particular, de modo que si se interpreta desde la óptica de la persona hace visible su pertenencia a un grupo, a una clase social, etc. Estos modelos retoman la teoría de la acción y la idea del precio como la cantidad de un bien que se recibe a cambio de otro; lo que según Appadurai (1986) y Thomas (1991) es una regresión a la visión económica. En ese contexto, Dumont (1982) argumenta que la noción de valor está relacionada con el concepto de jerarquía, aunque esta tesis se basa en el estudio de sociedades sumamente jerarquizadas, como la de castas en India. Dumont establece correspondencias dicotómicas binarias de conceptos para determinar las "relaciones entre relaciones", técnica que Graeber critica porque no tiene en cuenta el proceso de valoración de cada par ni las relaciones económicas asimétricas.

En esa misma década surgió un enfoque fenomenológico impulsado por Turner y Munn, que no se deriva directamente de Marx pero sigue la tradición dialéctica y se apoya en la teoría del valor trabajo, a la que reinterpreta equiparando el trabajo a la creatividad humana; de modo que el valor de las cosas se mide por las acciones necesarias para conseguirlas. Ya vimos que en Marx el precio era una expresión exacta del valor; y, además, no en todas las sociedades se puede valorar el trabajo en términos de mercado. En las sociedades tradicionales sin mercado, el valor es igual al de las acciones que representa, mientras que en las sociedades de mercado, el dinero es una representación simbólica del valor-trabajo de un producto.

Mencionemos por último a otros autores. Castaingts entiende el valor y la valoración como "hechos culturales", como un proceso simbólico, un aspecto que no tienen en cuenta el subjetivismo ni el objetivismo. Etzioni señala que el valor tiene dos fuentes: el placer y 
la moral; que refleja un acto moral basado en el autocontrol, mientras que la racionalización de la utilidad suprime los juicios de valor $(1988,101)$. Etzioni rompe con la concepción del homo ceconomicus y recalca que la elección no es unidimensional sino que ocurre en situaciones complejas que incluyen bases normativas y afectivas que determinan cómo se adquiere y se procesa la información. Cuando los valores se internalizan pasan a ser parte de la percepción de los individuos, y cuando son externos restringen la toma de decisiones. Bourdieu (1979), quien coincide con Veblen, vincula la idea de valor a la jerarquización social: los individuos tienden a considerar valiosos los bienes que consumen los grupos de estatus superior, que dan lugar a modelos de gusto y distinción que configuran un sistema clasificatorio de valores y estilos de vida. La distinción no solo jerarquiza los objetos -según grupos y clases sociales- sino que estos promueven la jerarquía, y suscitan conflictos y luchas de poder. A conclusiones parecidas llegan Girard a partir del concepto de "deseo mimético", Greimas a partir de la lucha por la construcción y apropiación de objetos calificantes, y Castaingts, quien resalta la lucha por la apropiación de la "acción simbólica" sobre todo entre las clases dominantes.

\section{SOBRE EL “VALOR”Y LA “UTILIDAD”}

La revisión de la literatura nos lleva a decir que el valor es una interpretación simbólica y el precio un resultado de la negociación entre partes, que son dos caras de un mismo fenómeno; una vista desde una perspectiva cualitativa y subjetiva, y la otra como una medida cuantitativa y objetiva. Esta dicotomía ha suscitado un prolongado debate, a veces estéril, entre autores y escuelas.

Para entender mejor esos conceptos es conveniente partir de la noción de "cosa útil", aquello que satisface necesidades humanas. Estas necesidades incluyen las puramente biológicas, asociadas a la supervivencia del individuo y de la especie; y las necesidades culturales, sociales y psicológicas, asociadas a la continuidad de la sociedad y sus instituciones. El tema de las necesidades ha sido tratado por distintas disciplinas, casi siempre clasificándolas, a veces de modo jerárquico, según lo que juzguen prioritario para la vida y el bienestar social. En economía se las suele clasificar distinguiendo entre bienes de primera necesidad y otros bienes, o según la mayor o menor inmediatez de la satisfacción que producen. En la antropología clásica, Malinowski argumentó que las instituciones culturales surgían para satisfacer dos tipos de necesidades: biológicas o primarias (nutrición, reproducción, seguridad, crecimiento, etc.) y psicológicas o secundarias. 
El hecho que enfrenta a las diferentes visiones teóricas y que condiciona la acción humana es que solo algunas cosas útiles se consideran bienes económicos que poseen valor o generan utilidad. Como el concepto de necesidad se relaciona con el de utilidad, la esfera de lo "útil" se extiende de lo objetivo a lo subjetivo y de lo individual a lo social. La cosa útil es un "bien" cuando se cumplen cuatro condiciones: que haya una necesidad humana, que la cosa útil la satisfaga, que el individuo perciba la relación causal entre la cosa y la satisfacción, y que se pueda disponer de la cosa. Los individuos estiman la cantidad requerida para satisfacer sus necesidades, que puede ser mayor o menor de la que se dispone. Cuando el bien es escaso con respecto a las necesidades, tiene un carácter económico o un valor. Si es abundante no es económico y carece de valor. $\mathrm{El}$ hecho de "no estar disponible" se puede deber a factores ecológicos, sociales o jurídicos. Los bienes son, pues, cosas útiles que poseen valor, y este es proporcional a su disponibilidad relativa.

El valor no es una propiedad intrínseca al bien sino una construcción social en proceso de cambio y redefinición, un hecho cultural que no se puede considerar objetivo y universal. Como construcción social, se puede representar en términos cuantitativos o de proporciones. Entre utilidad total y utilidad marginal hay diferencias más allá de las cuantitativas. Los valores preferenciales que aumentan la utilidad total están asociados a lo que la sociedad considera deseable y, dentro de ella, cada grupo o clase social. En cambio la utilidad marginal es siempre relativa y subjetiva, depende de las preferencias individuales. Al pasar de lo individual a lo colectivo, es posible que las preferencias sean similares -que muchas personas tengan las mismas expectativas y deseos- debido a influencias culturales y, en la actualidad, debido al marketing, la publicidad y otros medios. Esta coincidencia colectiva de las preferencias es lo que se conoce como valor de uso.

$\mathrm{El}$ "valor de cambio", el precio, se refiere al intercambio de bienes o servicios, a la cantidad de un bien que se recibe a cambio de otro. La antropología aporta una visión transcultural del intercambio que no se limita al "valor de cambio", y muestra que su fin no es siempre maximizar el beneficio. E1 precio solo es resultado del intercambio inmediato en sociedades de mercado, casi siempre en términos de dinero. El precio implica una valoración racional, no necesariamente equilibrada, es decir, los bienes y servicios que se intercambian no necesariamente tienen el mismo "valor" para ambas partes. En la fijación del precio, las partes que intercambian buscan satisfacer sus necesidades considerando sus preferencias, y entran en una negocia- 
ción en la que intentan cumplir sus expectativas y llegan a un acuerdo que se plasma en el precio de mercado.

\section{CONCLUSIONES}

La economía y la antropología social se han desconocido mutuamente, en gran medida debido a sus grandes diferencias metodológicas, pero no solo a ellas. La economía da prioridad al comportamiento individual, que en el agregado configura tipos y estructuras sociales; 1a antropología inicialmente dio prioridad al comportamiento colectivo o social. Pero en los últimos años ha habido un acercamiento a través del pensamiento institucionalista, que intenta superar la visión económica formalista, nutriéndose de otras ciencias sociales. La separación entre economía y antropología, el divorcio, como lo llamó Pearson (2000, 982), poco a poco lleva a una reconciliación metodológica y teórica que da mayor realismo a los modelos económicos, pues al incluir las instituciones interpretan mejor la complejidad del mundo económico.

Nuestra revisión de las ideas acerca del valor muestra que las discrepancias entre el objetivismo y el subjetivismo son aparentes. La sucesión de doctrinas y su vigencia total o parcial diferencian a la economía de otras disciplinas y la convierten, como señaló Myrdal, en algo similar a la teología. El debate entre objetivistas y subjetivistas en economía es tan estéril como el debate entre sustantivistas y formalistas en antropología. La dimensión cultural (instituciones, normas, costumbres y hábitos) es esencial en la determinación del valor social de los bienes, en el que convergen el valor económico y el valor simbólico cultural. El valor de uso no es un fenómeno limitado estrictamente a la esfera individual, sino que depende de la cultura imperante, que propone o impone pautas colectivas.

\section{REFERENCIAS BIBLIOGRÁFICAS}

1. Appadurai, A. The social life of things: Commodities in cultural perspective, Cambridge, Cambridge University Press, 1986.

2. Ayres, C. E. The theory of economic progress, Chapel Hill, University of North Carolina Press, 1944.

3. Bardhan, P., ed. Conversations between economists and anthropologists: Methodological issues in measuring economic change in rural India, Nueva Delhi, Oxford University Press, 1989.

4. Bardhan, P. e I. Ray. "Methodological approaches in economics and anthropology", J. Davis y W. Dofsma, eds., The Elgar Companion to Social Economics, Cheltenham, Elgar Publishing, 2008, pp. 427-456.

5. Blaug, M. The methodology of economics [1980], Nueva York, Cambridge University Press, 1992. 
6. Böhm-Bawerk, E. von. Capital and interest, 3 vols. [1921], South Holland, Libertarian Press, 1959.

7. Booyens, J. "Economics and Anthropology: in search of partnerships", South African Journal of Economics, 66, 4, 1998, pp. 585-596.

8. Bourdieu, P. La distinction. Critique social du jugement, París, Editions de Minuit, 1979.

9. Buckley, P. J. y M. Chapman. "Economics and social anthropology Reconciling difference”, Human Relations 49, 9, 1996, pp. 1123-1150.

10. Cantillon, R. Ensayo sobre la naturaleza del comercio en general [1755], México, D. F., Fondo de Cultura Económica, 1950.

11. Castaingts, J. Simbolismos del dinero. Antropología y economía: una encrucijada, Barcelona-Iztapalapa, Anthropos, 2002.

12. Cohen, P. S. "Análisis económico y hombre económico. Comentarios sobre una controversia”, R. Firth, ed., Temas de antropología económica, México, D. F., Fondo de Cultura Económica, 1974, pp. 95-121.

13. Dalton, G. "Economic theory and primitive society", American Anthropologist 63, 1, 1961, pp. 1-25.

14. Dalton, G. "Is economics anthropology of interest to economists?", American Economics Review 68, 2, 1978, pp. 23-27.

15. Devons, E. y M. Gluckman. "Conclusion: Modes and consequences of limiting a field of study", M. Gluckman, ed., Closed systems and open minds: The limits of naivety in social anthropology, Edimburgo, Oliver \& Boyd, 1964, pp. 158-261.

16. Díaz de R., A. "Valer y valor. Una exhumación de la teoría del valor para reflexionar sobre la desigualdad y la diferencia en relación con la escuela", Revista de Antropología Social 16, 2007, pp. 117-158.

17. Dobb, M. Political economy and capitalism: Some essays in economic tradition, Londres, Routledge, 1937.

18. Douglas, M. “The exclusion of economics”, Times Literary Suppl., 6 de julio de 1973, pp. 781-782.

19. Dumont, L. “On value", Proceedings of the British Academy 66, 1982, pp. 207-241.

20. Edgeworth, F. Y. Mathematical psychics. An essay on the application of mathematics to moral sciences, Londres, Kegan Paul \& Co., 1881.

21. Ensminger, J. "Experimental economics: A powerful new method for theory testing in anthropology", J. Ensminger, ed., Theory in economic anthropology, Walnut Creek, Altamira Press, 2002, pp. 59-78.

22. Etzioni, A. The moral dimension. Toward a new economics, Nueva York, The Free Press, 1988.

23. Firth, R. Primitive economics of the New Zealand Maoris, Londres, Routledge, 1929.

24. Foster, R. J. "The uses of use value: Marketing, value creation, and the exigencies of consumption work", D. Zwick y J. Cayla, eds., Inside marketing: Practices, ideologies, devices, Oxford, Oxford Scholarship, 2011, pp. 42-57.

25. Garnett, R. F. "Postmodernism and theories of value: New grounds for Institutionalist/Marxist dialogue?”, Journal of Economic Issues 33, 4, 1999, pp. 817-834. 
26. Genovesi, A. Lecciones de comercio o bien de economía civil [1769], t. III, Madrid, Viuda de Ibarra, 1786.

27. Girard, R. La violence et le sacré, París, Grasset, Pluriel, 1972.

28. Godelier, M. Instituciones económicas, Barcelona, Anagrama, 1981.

29. Graeber, D. Toward an anthropological theory of value, Nueva York, Palgrave, 2001.

30. Graeber, D. "Value: Anthropological theories of value”, J. G. Carrier, ed., A Handbook of Economic Anthropology, Northampton, Elgar Publishing, 2006, pp. 439-454.

31. Gregory, C. A. Savage money: The anthropology and politics of commodity exchange, Amsterdam, Harwood Academic Publishers, 1997.

32. Haddad, L.; J. Hoddinott y H. Alderman. Intra-household resource allocation in developing countries. Models, methods, and policy, Baltimore, Johns Hopkins University Press, 1997.

33. Hamilton, D. "The great wheel of wealth. A reflection of social reciprocity", Journal of Economics and Sociology 24, julio de 1965, pp. 241-248.

34. Hamilton, D. "The meaning of anthropology for economic science: A case for intellectual reciprocity", Journal of Economic Issues 25, 4, 1991, pp. 937-949.

35. Harriss, J. “The case for cross-disciplinary approaches in international development”, World Development 30, 3, 2002, pp. 487-496.

36. Henrich, J. et al. Foundations of human sociality: Economic experiments and ethnographic evidence from fifteen small-scale societies, Nueva York, Oxford University Press, 2004.

37. Herskovits, M. J. Economic anthropology. A study in comparative economics, Nueva York, Alfred A. Knopf, 1952.

38. Hicks, J. R. Value and capital. An inquiry into some fundamental principles of economic theory, Oxford, Oxford University Press, 1939.

39. Hicks, J. R. y R. G. Allen. "A reconsideration of the theory of value, Part I", Economica 1, 1, 1934, pp. 52-76.

40. Jevons, W. S. The theory of political economy, Londres, McMillan, 1871.

41. Keynes, J. M. Teoría general del empleo, el interés y el dinero [1936], Madrid, Ediciones Aosta, 1998.

42. Kluckhohn, C. "Values and value-orientations in the theory of action. An exploration in definition and classification", T. Parsons y E. Shils, eds., Toward a general theory of action, Cambridge, Cambridge University Press, 1951, pp. 388-433.

43. Knight, F. H. "Anthropology and economics", Journal of Political Economy 49, 1941, pp. 247-268.

44. Kurz, H. D. y N. Mondadori, "Sraffa and the labour theory of value: A few observations”, J. Vint, J. S. Metcalfe et al., eds., Economic theory and economic thought: Essays in honour of Ian Steedman, Abigdon, Routledge, 2010, pp. 189-215.

45. Lancaster, K. J. "A new approach to consumer theory", Journal of Political Economy LXXIV, 2, 1966, pp. 132-157.

46. Lipton, M. "Economics and anthropology: Grounding models in relationships”, World Development 20,10, 1992, pp. 1541-1546. 
47. Locke, J. Segundo tratado sobre el gobierno civil [1689], Madrid, Alianza Editorial, 2000.

48. Malinowski, B. Argonauts of the Western Pacific, Londres, Routledge, 1922.

49. Malthus, T. R. Principios de economía política [1820], México, D. F., Fondo de Cultura Económica, 1946.

50. Marshall, A. Principles of economics, Londres, Macmillan, 1890.

51. Marx, K. El capital [1867], vol. I, Barcelona, Orbis, 1988.

52. Menger, C. Grundsätze der Volkswirtschaftslebre, Viena, Wilhelm Braumüller, 1871.

53. Mill, J. S. "On the definition of political economy and the method of investigation proper to it", J. M. Robson, ed., Collected works of John Stuart Mill [1836], vol. 4, Toronto, University of Toronto Press, 1987.

54. Mill, J. S. Principios de economía politica [1848], México, D. F., Fondo de Cultura Económica, 1951.

55. Mises, L. von. La acción humana. Tratado de economia [1949], Madrid, Unión editorial, 1980.

56. Mishan, E. J. "Theories of consumer's behaviour: A cynical view”, Economica 28, 109, 1961, pp. 1-11.

57. Morris, C. Varieties of human value, Chicago, University of Chicago Press, 1956.

58. North, D. Institutions, institutional change and economic performance, Cambridge, Cambridge University Press, 1990.

59. Pareto, W. Manual de economía politica [1906], Madrid, Atalaya, 1945.

60. Pearson, H. "Homo economicus goes native, 1859-1945: The rise and fall of primitive economics", History of Political Economy 32, 4, 2000, pp. 933-989.

61. Pearson, H. "Primitive economics: A reply", History of Political Economy 34, 1, 2002, pp. 273-281.

62. Perry C. M. "Value as any object of any interest", International Journal of Ethics 40, 4, 1930, pp. 490-495.

63. Perry, R. B. General theory of value. Its meaning and basic principles construed in terms of interest [1926], Cambridge, Mass., Harvard University Press, 1950.

64. Pieters, R. y H. Baumgartner. "Who talks to whom? Intra- and interdisciplinary communication of economics journal", Journals of Economics Literature 40, 2, 2002, pp. 483-509.

65. Pryor, F. L. The origins of the economy: A comparative study of distribution in primitive and peasant economies, Nueva York, Academic Press, 1977.

66. Rao, V. y M. Walton. Culture and public action, Stanford, Ca., Stanford University Press, 2004.

67. Ricardo, D. Principios de economía politica y tributación [1821], México, D. F., Fondo de Cultura Económica, 2004.

68. Samuelson, P. A. "A note on the pure theory of consumer's behaviour", Economica 5 17, 1938, pp. 61-71.

69. Say, J. B. Tratado de economía política [1803], México, D. F., Fondo de Cultura Económica, 2000.

70. Sen, A. "Sraffa, Wittgenstein, and Gramsci", Journal of Economic Literature 41, 4, 2003, pp. 1240-1255. 
71. Smith, A. Investigación de la naturaleza y causa de la riqueza de las naciones [1776], Valladolid, Oficina Viuda e hijos de Santander, 1794.

72. Thomas, N. Entangled objects: Exchange material culture, and colonialism in the Pacific, Cambridge, Mass., Harvard University Press, 1991.

73. Tool, M. R. "A social value theory in neoinstitutional economics", Journal of Economic Issues 11, 4, 1977, pp. 823-844.

74. Veblen, T. "The limitations of marginal utility", Journal of Political Economy 17, 9, 1909, pp. 620-636.

75. Veblen, T. The theory of leisure class: An economic study of institutions [1899], Macmillan, Nueva York, 1915.

76. Verri, P. Tratado elemental de la economía politica [1771], Madrid, Imprenta de Espinosa, 1820.

77. Walras, L. Elements of pure economics or the theory of social wealth [1874], Fairfield, Augustus Kelley Publishers, 1977.

78. White, H. "Combining quantitative and qualitative approaches in poverty analysis”, World Development 30, 3, 2002, pp. 511-522. 